\title{
Ionospheric Effects of Electrostatic Fields Generated in the Outer Magnetosphere
}

\author{
George C. Reid \\ Central Radio Propagation Laboratory, National Bureau of Standards, Boulder, Colo.
}

(Received January 21, 1965)

\begin{abstract}
The conduction of electrostatic fields from the outer magnetosphere to the ionosphere via the geomagnetic field lines is studied in a quantitative fashion. It is assumed that any arbitrary pattern of turbulent fields in the magnetosphere can be analyzed into Fourier components, and the transfer of components whose wavelength lies between $0.1 \mathrm{~km}$ and $1000 \mathrm{~km}$ (as measured in the ionosphere) is examined. It is concluded that components of wavelength less than about $1 \mathrm{~km}$ will be severely attenuated before reaching the $F$ region, whereas components with wavelength greater than about $10 \mathrm{~km}$ will be only slightly attenuated. The application of these results to the theory of the origin of ionospheric irregularities is discussed.

The transfer of these electrostatic fields must necessarily be accompanied by the appearance of weak electrostatic fields along the direction of the magnetic field. The size of these fields is estimated, and their possible effect on the acceleration of electrons is discussed.
\end{abstract}

\section{Introduction}

The close relationship between the solar wind and the various types of geophysical activity peculiar to high latitudes, including the aurora, is now fairly well established. As a consequence, it seems reasonable to look for the basic cause of these effects in the dynamical interaction of the solar wind with the outer reaches of the earth's magnetosphere. Many of the consequences of this interaction have been outlined by Axford and Hines [1961] who suggested the existence of a largescale convection of the material of the outer magnetosphere driven by viscous forces at the interface between the magnetosphere and the domain of the solar wind. Because of the high electrical conductivity of the magnetospheric plasma, the magnetic field is frozen to the material, and the convective flow pattern can be thought of in hydromagnetic terms as a steady motion of the lines of force throughout the magnetosphere and the major portion of the ionosphere. Alternatively, the motion can be described by saying that the motion of the material in the outer magnetosphere sets up an electrostatic field which exactly cancels the $\mathbf{v} \times \mathbf{B}$ dynamo field, thus allowing the material to flow freely. Because of the high conductivity along the direction of the magnetic field, the field lines are very nearly equipotentials of the electrostatic field, and the field is transferred down into the ionosphere, there driving the ionization in the direction of $\mathbf{E} \times \mathbf{B}$. The exact equivalence of these two representations of the motions is a well-known result of the hydromagnetic theory of highly ionized plasmas, and is discussed in some detail by Axford and Hines [1961] and Hines [1963].

An important feature of the Axford-Hines theory is the suggestion that the outer magnetosphere is 'turbulent', i.e., that perturbations of either $v$ or $B$ (or both) exist, and that much of the spatial finestructure observed in high-latitude geophysical phenomena is due to the inward convection of this turbulence and its intensification by adiabatic compression of the tubes of force. There should be a corresponding turbulent component of the electrostatic field appearing in the ionosphere, and this might lead to the formation of field-alined irregularities in ionization such as those giving rise to spread- $F$ ionosonde echoes, scintillation of radio-star signals, and auroral radar echoes.

The mechanism is thus somewhat similar to that proposed by Dagg [1957] to account for the formation of $F$-region irregularities. He suggested that the upward transfer of turbulent electrostatic fields generated in the dynamo region of the ionosphere might lead to the formation of irregularities at much greater heights, and his proposal was examined in detail and criticized by Farley [1959, 1960, 1961], Dougherty [1959] and Spreiter and Briggs [1961]. The general conclusion was that turbulent fields of the appropriate scale-sizes would indeed be transferred to the required altitudes, but that they would lead to the formation of irregularities only in the presence of an existing large-scale gradient of electron density, since the drift velocity in the $F$ region must be very nearly divergence-free. Stated in another way, the random component of the electrostatic field will lead to a permutation of the tubes of force, but will lead to the appearance of irregularities only if the permuting tubes originally contained different amounts of ionization and are thus distinguishable from one another after permutation. The mechanism of Axford and Hines [1961] must operate under similar restric- 
tions, and in discussing irregularities here we shall implicitly assume that the necessary large-scale gradients of ionization do in fact exist. The suggestion is then critically dependent on the efficiency with which small-scale electrostatic fields can be mapped down the field lines from the magnetosphere to the ionosphere, and in this paper we attempt to examine this process quantitatively. One important consequence of the analysis to be described is the fact that the existence of turbulence in the magnetosphere must necessarily lead to the existence of weak electrostatic fields along the direction of the magnetic field in a steady state. Some of the consequences of the existence of these fields will be discussed in a later section.

\section{Theory of Electrostatic-Field Mapping}

The geomagnetic field gives rise to a pronounced anisotropy in the electrical properties of the ionospheric plasma, and the conductivity becomes a tensor quantity, i.e., an electric field vector $\mathbf{E}$ in a given direction gives rise to a current density vector $\mathbf{j}$ in $\mathbf{a}$ different direction. The anisotropy is most easily treated if we adopt a rectangular set of axes, one of which is along the local direction of the magnetic field vector, B. We shall assume here that $\mathbf{B}$ lies along the positive $z$-axis, and that the $z$-axis is directed vertically downward in the northern hemisphere. The current density is then given by (e.g., Baker and Martyn, 1953)

$\mathbf{j}=\frac{\sigma_{0}}{B^{2}}(\mathbf{E} \cdot \mathbf{B}) \mathbf{B}+\frac{\sigma_{1}}{B^{2}}\{\mathbf{B} \times(\mathbf{E} \times \mathbf{B})\}+\frac{\sigma_{2}}{B}(\mathbf{B} \times \mathbf{E})$,

where $\sigma_{0}, \sigma_{1}, \sigma_{2}$ are respectively the longitudinal conductivity (along the direction of $\mathbf{B}$ ), the Pedersen conductivity (in the plane of $\mathbf{E}$ and $\mathbf{B}$ and normal to $\mathbf{B}$ ), and the Hall conductivity (normal to both $\mathbf{E}$ and $\mathbf{B}$ ). Thus we have

$$
\begin{aligned}
& j_{x}=\sigma_{1} E_{x}-\sigma_{2} E_{y} \\
& j_{y}=\sigma_{1} E_{y}+\sigma_{2} E_{x} \\
& j_{z}=\sigma_{0} E_{z} .
\end{aligned}
$$

Since we are concerned with electrostatic fields, we can write

$$
\mathbf{E}=-\operatorname{grad} \varphi
$$

where $\varphi$ is a scalar potential, and in a steady state we assume

$$
\operatorname{div} \mathbf{j}=0 \text {. }
$$

Substituting (2) and (3) in (4), we find

$$
\frac{\partial^{2} \varphi}{\partial x^{2}}+\frac{\partial^{2} \varphi}{\partial y^{2}}+\frac{1}{\sigma_{1}} \frac{\partial}{\partial z}\left(\sigma_{0} \frac{\partial \varphi}{\partial z}\right)=0
$$

if we assume that the three conductivities vary only in the vertical direction. The Hall conductivity, $\sigma_{2}$, does not appear in this equation, since our assumptions of a vertical magnetic field and a uniform ionosphere in the horizontal plane allow the Hall current to flow in uninterrupted divergence-free loops around the direction of the magnetic field, thus playing no part in determining the overall zero-divergence condition for the current flow.

The problem is formally similar to that encountered in the calculation of electromagnetic fields in ground of inhomogeneous and anisotropic conductivity [e.g., Smythe, 1939; Wait and Conda, 1958] and was first treated for the case for the ionosphere by Farley [1959, 1960]. We essentially follow the treatment of the latter author, who investigated the transfer of electrostatic fields from the dynamo region of the ionosphere upward into the $F$ region. In this approach the strong anisotropy in the conductivity is treated by a transformation of the coordinate system from $(x, y, z)$ to a new system $(\xi, \eta, \zeta)$, defined by

$$
\begin{gathered}
\xi=x \\
\eta=y \\
d \zeta=\left(\sigma_{1} / \sigma_{0}\right)^{1 / 2} d z .
\end{gathered}
$$

Applying this transformation to (5), we find

$$
\frac{\partial^{2} \varphi}{\partial \xi^{2}}+\frac{\partial^{2} \varphi}{\partial \eta^{2}}+\frac{\partial^{2} \varphi}{\partial \zeta^{2}}+\frac{\partial \varphi}{\partial \zeta} \frac{\partial}{\partial \zeta}\left(\ln \sigma_{m}\right)=0
$$

where

$$
\sigma_{m}=\left(\sigma_{0} \sigma_{1}\right)^{1 / 2} .
$$

This equation is greatly simplified if we assume that $\sigma_{m}$ can be represented, at least over limited intervals of height, by the expression

$$
\sigma_{m}=K \exp (-\rho \zeta)
$$

Then (7) becomes

$$
\frac{\partial^{2} \varphi}{\partial \xi^{2}}+\frac{\partial^{2} \varphi}{\partial \eta^{2}}+\frac{\partial^{2} \varphi}{\partial \zeta^{2}}-\rho \frac{\partial \varphi}{\partial \zeta}=0
$$

We now assume that the electrostatic potential is generated at a 'source level', $\zeta_{0}$, and has a sinusoidal spatial variation with a wavelength $\lambda^{-}$in both the $\xi$ - and $\eta$-directions. The sinusoidal variation can be regarded as one Fourier component of an arbitrary distribution, and the assumption that the spatial wavelength is the same in both directions is introduced for convenience and does not affect the conclusions materially. Under these conditions, (10) is satisfied by

$$
\varphi=\varphi_{0} \exp \left[A\left(\zeta-\zeta_{0}\right)\right] \exp \left[\frac{2 \pi i}{\lambda}(\xi+\eta)\right],
$$


where $A$ is a root of the quadratic equation

$$
A^{2}-\rho A-\frac{8 \pi^{2}}{\lambda^{2}}=0
$$

i.e.,

$$
A=\frac{\rho}{2} \pm\left[\frac{\rho^{2}}{4}+\frac{8 \pi^{2}}{\lambda^{2}}\right]^{1 / 2}
$$

Let these two roots be denoted by

$$
\alpha=\frac{\rho}{2}-\left[\frac{\rho^{2}}{4}+\frac{8 \pi^{2}}{\lambda^{2}}\right]^{1 / 2} \quad \beta=\frac{\rho}{2}+\left[\frac{\rho^{2}}{4}+\frac{8 \pi^{2}}{\lambda^{2}}\right]^{1 / 2} .
$$

The complete solution of (10) is then given by

$$
\begin{array}{r}
\varphi=\varphi_{0}\left[P \exp \left\{\alpha\left(\zeta-\zeta_{0}\right)\right\}+Q \exp \left\{\beta\left(\zeta-\zeta_{0}\right)\right\}\right] \\
\exp \left[\frac{2 \pi i}{\lambda}(\xi+\eta)\right] .
\end{array}
$$

In order to make a close approximation to physical conditions, we divide the ionosphere below $\zeta_{0}$ into $n$ slabs, in each of which the conductivity $\sigma_{m}$ is assumed to obey (9) and is continuous across the interfaces between the slabs. Any actual profile of conductivity can then be used by adopting a sufficiently large value for $n$. The $m$ th slab then lies between $\zeta_{m-1}$ and $\zeta_{m}$, and the $n$th slab extends from $\zeta_{n-1}$ to infinity. Let the values of the coefficients in the $m$ th slab be denoted by $\alpha_{m}, \beta_{m}, P_{m}, Q_{m}$. In the $m$ th slab the potential then satisfies

$$
\begin{aligned}
\varphi=\varphi_{0}\left[P_{m} \exp \left\{\alpha_{m}\left(\zeta-\zeta_{m-1}\right)\right\}\right. & \left.+Q_{m} \exp \left\{\beta_{m}\left(\zeta-\zeta_{m-1}\right)\right\}\right] \\
& \exp \left[\frac{2 \pi i}{\lambda}(\xi+\eta)\right] .
\end{aligned}
$$

Our boundary condition at the source level then leads to

$$
P_{1}+Q_{1}=1 \text {. }
$$

At each of the $(n-1)$ interfaces between slabs the potential, $\varphi$, and its vertical derivative, $\partial \varphi / \partial \zeta$, should be continuous. Thus at the interface between the $m$ th and $(m+1)$ th slabs we have

$$
\begin{array}{r}
P_{m} \exp \left\{\alpha_{m}\left(\zeta_{m}-\zeta_{m-1}\right)\right\}+Q_{m} \exp \left\{\beta_{m}\left(\zeta_{m}-\zeta_{m-1}\right)\right\} \\
=P_{m+1}+Q_{m+1}
\end{array}
$$

$$
\alpha_{m} P_{m} \exp \left\{\alpha_{m}\left(\zeta_{m}-\zeta_{m-1}\right)\right\}+\beta_{m} Q_{m} \exp \left\{\beta_{m}\left(\zeta_{m}-\zeta_{m-1}\right)\right\}
$$

$$
=\alpha_{m+1} P_{m+1}+\beta_{m+1} Q_{m+1} \text {. }
$$

The $(n-1)$ interfaces yield $(2 n-2)$ equations; with the addition of (17) we have $(2 n-1)$ equations, and $2 n$ unknowns (the values of $P_{m}$ and $Q_{m}$ in the $n$ slabs). The final equation is provided by adopting a boundary condition at infinity, the base of the $n$th slab. We assume that as $\zeta \rightarrow \infty$ the potential vanishes. Farley $[1959,1960]$ also applied this boundary condition and was criticized by Spreiter and Briggs [1961] [see also Farley 1961] on the grounds that the conductivity of the ionosphere at great heights $(\zeta \rightarrow \infty$ in Farley's model) is such that a vanishing potential leads to a finite current at infinity. The latter authors obtained solutions of (10) with the more realistic boundary condition of vanishing current at infinity. In the problem considered by both Farley and Spreiter and Briggs, however, the direction of the positive $\zeta$-axis was upward from the earth; in our case, the $\zeta$-axis points downward, and the fact that the conductivity decreases rapidly to zero below the ionosphere leads to the disappearance of the current as well as the potential at infinity. Since $\alpha_{n}$ must be negative and $\beta_{n}$ must be positive, this boundary condition leads to an equation

$$
Q_{n}=0 \text {. }
$$

The $2 n$ equations given by (17), (18), and (19) now enable us to find the $2 n$ unknown coefficients $P_{m}$, $Q_{m}(m=1, n)$.

Having solved the equations, the "transmission factor', $Z$, for the electrostatic potential can be found at any height from

$$
\begin{array}{r}
Z=\varphi / \varphi_{\text {source }}=P_{m} \exp \left\{\alpha_{m}\left(\zeta-\zeta_{m-1}\right)\right\}+Q_{m} \exp \\
\left\{\beta_{m}\left(\zeta-\zeta_{m-1}\right)\right\} .
\end{array}
$$

This is equal to the transmission factor for the horizontal electrostatic field, since

$\left(\frac{\partial \varphi}{\partial \xi}\right) /\left(\frac{\partial \varphi}{\partial \xi}\right)_{\text {source }}=\left(\frac{\partial \varphi}{\partial \eta}\right) /\left(\frac{\partial \varphi}{\partial \eta}\right)_{\text {source }}=\varphi / \varphi_{\text {source }}$

The vertical component of the electrostatic field, along the direction of the magnetic field, is given by

$$
\begin{aligned}
& E_{z}=-\left(\frac{\sigma_{1}}{\sigma_{0}}\right)^{1 / 2} \frac{\partial \varphi}{\partial \zeta}=-\left(\frac{\sigma_{1}}{\sigma_{0}}\right)^{1 / 2} \varphi_{0}\left[\alpha_{m} P_{m} \exp \left\{\alpha_{m}\left(\zeta-\zeta_{m-1}\right)\right\}\right. \\
& \left.+\beta_{m} Q_{m} \exp \left\{\beta_{m}\left(\zeta-\zeta_{m-1}\right)\right\}\right] \exp \left[\frac{2 \pi i}{\lambda}(\xi+\eta)\right] .
\end{aligned}
$$

We shall denote by $L$ the magnitude of the ratio of the longitudinal component of the electrostatic field to the source potential at the top of the magnetic field line, i.e.,

$$
\begin{array}{r}
L=\left(\frac{\sigma_{1}}{\sigma_{0}}\right)^{1 / 2}\left[\alpha_{m} P_{m} \exp \left\{\alpha_{m}\left(\zeta-\zeta_{m-1}\right)\right\}+\beta_{m} Q_{m}\right. \\
\left.\cdot \exp \left\{\beta_{m}\left(\zeta-\zeta_{m-1}\right)\right\}\right] .
\end{array}
$$

It is worth noting that $L$ is a maximum along the field line with the maximum source potential, whereas the horizontal field is a maximum where the gradient of the source potential is a maximum. Physically, this is due to the fact that the longitudinal field arises 
from the necessity of supplying current along the magnetic field to account for the horizontal variation of Pedersen current, and the latter is a maximum where the transverse field is varying most rapidly, not where it has its maximum value.

\section{Ionospheric Conductivities}

The conductivities $\sigma_{0}$ and $\sigma_{1}$ are functions of several quantities whose values can as yet only be estimated. The theory of the electrical conductivity of the ionosphere below the peak of the $F 2$ region has been reviewed by Chapman [1956], using as a basis the work of Chapman and Cowling [1939] and Cowling [1945]. For our purpose, we shall assume that the conductivities are given to a sufficiently good approximation by simple mean-free-path theory. The longitudinal conductivity, $\sigma_{0}$, is the same as the conductivity in the absence of a magnetic field, and is given by

$$
\sigma_{0}=N e^{2}\left[\frac{1}{m_{e} \nu_{e}}+\frac{1}{m_{i} \nu_{i}}\right],
$$

in which the subscripts $e$ and $i$ refer to electrons and ions respectively, $N$ is the number density of ions or electrons, $m$ and $\nu$ are the respective masses and collision frequencies, and $e$ is the electronic charge. We shall use MKSA units, in which $e$ is measured in coulombs and the conductivities in mhos per meter. The electron collision frequency, $\nu_{e}$, is assumed to contain contributions from both electron-neutral $\left(\nu_{e n}\right)$ and electron-ion $\left(\nu_{e i}\right)$ collisions, i.e.,

$$
\nu_{e}=\nu_{e n}+\nu_{e i}
$$

whereas $\nu_{i}$ contains only a contribution from ionneutral collisions, since the ions are almost unaffected by collisions with the much lighter electrons.

Chapman [1956] derives the following effective values for the collision frequencies (converted to MKSA units)

$$
\begin{gathered}
\nu_{i}=2.6 \times 10^{-15}\left(N+n_{n}\right) W^{-1 / 2} \\
\nu_{e n}=5.4 \times 10^{-16} n_{n} T^{1 / 2} \\
\nu_{e i}=4.2 \times 10^{-6}\left\{14.1+\log _{10}\left(T^{3} / N\right)\right\} N T^{-3 / 2},
\end{gathered}
$$

where $W$ is the molecular weight of the ions, $T$ is the temperature, and the subscript $n$ refers to the neutral particles.

The Pedersen conductivity, $\sigma_{1}$, is given by the meanfree-path theory as

$$
\sigma_{1}=N e^{2}\left\{\frac{\nu_{e}}{m_{e}\left(\nu_{e}^{2}+\omega_{e}^{2}\right)}+\frac{\nu_{i}}{m_{i}\left(\nu_{i}^{2}+\omega_{i}^{2}\right)}\right\},
$$

where $\omega_{e}$ and $\omega_{i}$ are respectively the electron- and ion-gyrofrequencies, i.e.,

$$
\omega_{e, i}=\frac{e B}{m_{e, i}} .
$$

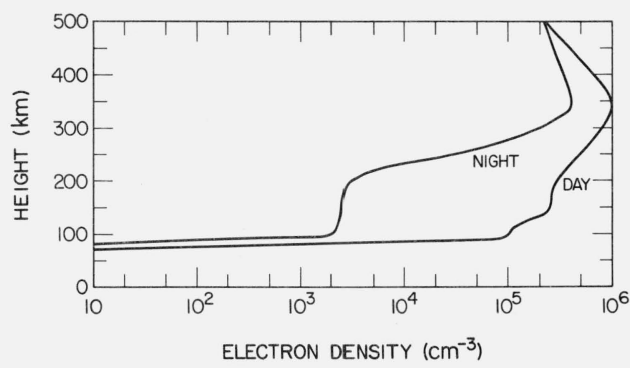

FIGURE 1. Electron-density profiles adopted for daytime and nighttime conditions below $500 \mathrm{~km}$ altitude.

Both conductivities depend on the electron density, both directly and through the electron-ion collision frequency as given by (28). For the purposes of this study we have adopted two profiles of electron density below $500 \mathrm{~km}$, one appropriate to daytime and one to nighttime conditions. These are shown in figure 1 .

The conductivity relations given above are applicable only to regions in which there are many more neutral particles than ions and electrons (though $\nu_{e n}$ is not necessarily large compared with $\nu_{e i}$ ). At altitudes well above the peak of the $F 2$ region this is no longer the case, and the conductivities approach those of a fully ionized plasma. Under these conditions, Chapman [1956] shows that the longitudinal conductivity is given by

$$
\sigma_{0}=N e^{2} / m_{e} \nu_{e i}
$$

and the Pedersen conductivity by

$$
\sigma_{1}=\sigma_{0} \frac{\nu_{e i}^{2}}{\nu_{e i}^{2}+\omega_{e}^{2}}
$$

Substituting from (28) and (30), we find

$$
\begin{gathered}
\sigma_{0}=\frac{2.4 \times 10^{5} e^{2} T^{3 / 2}}{m_{e}\left\{14.1+\log _{10}\left(T^{3} / N\right)\right\}} \\
\sigma_{1}=\frac{4.2 \times 10^{-6} m_{e}}{T^{3 / 2}}\left(\frac{N}{B}\right)^{2}\left\{14.1+\log _{10}\left(T^{3} / N\right)\right\},
\end{gathered}
$$

since at great altitudes $\nu_{e i}^{2} \ll \omega_{e}^{2}$.

In the present analysis we are not concerned with the values of the conductivities individually, but with their geometric mean, $\sigma_{m}$, and with the depth-scaling factor $\left(\sigma_{1} / \sigma_{0}\right)^{1 / 2}$. In the magnetosphere these are

$$
\sigma_{m}=e \frac{N}{B}
$$

and

$$
\begin{array}{r}
\left(\sigma_{1} / \sigma_{0}\right)^{1 / 2}=\frac{4.2 \times 10^{-6} m_{e}}{e T^{3 / 2}}\left(\frac{N}{B}\right)\left\{14.1+\log _{10}\left(T^{3} / N\right)\right\} \\
\left(=\nu_{e i} / \omega_{e}\right) .
\end{array}
$$




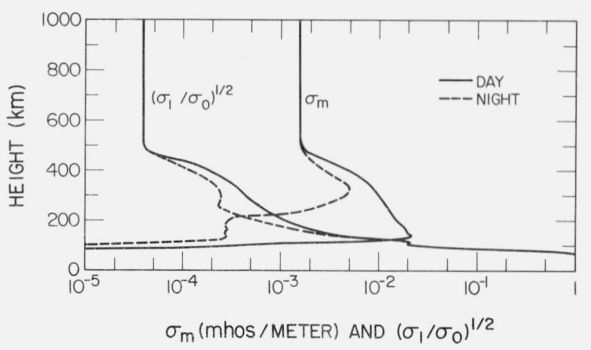

Figure 2. Height profiles of the geometric-mean conductivity $\left(\sigma_{m}\right)$ and the depth-scaling factor $\left(\sigma_{1} / \sigma_{0}\right)^{1 / 2}$ for daytime and nighttime conditions.

Both quantities are assumed to remain constant for all heights above $500 \mathrm{~km}$.

In both of these expressions, the height variation appears solely in the factor $(N / B)$, if we ignore the slow variation caused by the logarithmic term in (36), and assume an isothermal magnetosphere. Data obtained from measurements of nose-whistlers, however [Smith, 1961], have indicated that the ambient electron density in the magnetosphere is approximately proportional to the magnetic field strength, and the quantity $(N / B)$ is thus approximately independent of height. In the calculations that follow, we have adopted a model for the magnetosphere in which (35) and (36) are applicable from the source height down to an altitude of $500 \mathrm{~km}$; this is not strictly justifiable, but the likely departures from this height variation should not affect the conclusions materially. The value of $\rho$ in this height range (see eq (9)) is then zero. If we assume that at an altitude where $B$ is $10^{-7}$ $\mathrm{Wb} / \mathrm{m}^{-2}$ (100 gammas), $T$ is $2000{ }^{\circ} \mathrm{K}$, and $N$ is $10^{9}$ $\mathrm{m}^{-3}$, we find that $\left(\sigma_{1} / \sigma_{0}\right)^{1 / 2}$ is about $4 \times 10^{-5}$. In the analysis to be described, this value has been adopted for all altitudes in excess of $500 \mathrm{~km}$. The resultant models for $\sigma_{m}$ and $\left(\sigma_{1} / \sigma_{0}\right)^{1 / 2}$ are shown in figure 2 .

\section{Conditions in the Magnetosphere}

We have shown above that conditions in the magnetosphere (here defined rather arbitrarily as the region above $500 \mathrm{~km}$ ) are relatively simple from the conductivity point of view, since both $\sigma_{m}$ and $\left(\sigma_{1} / \sigma_{0}\right)^{1 / 2}$ can be regarded as constant with altitude to a first approximation. An additional complication is introduced in this region, however, by the rapid divergence of the field lines with increasing altitude. The model we have been discussing assumed that the magnetic field lines were parallel straight lines, a justifiable approximation within the ionosphere. The divergence of the field lines in the magnetosphere introduces two complications in the analysis. First, the spatial wavelength, $\lambda$, increases continuously with increasing altitude, and second, the area through which a given current must flow along the magnetic field lines increases continuously with altitude, leading to a decrease in the current density, $\mathbf{j}$, with altitude for a constant total current.
It can readily be shown that the separation between field lines in a dipole field is given by [e.g., Akasofu and Chapman, 1961].

$$
\begin{gathered}
S_{x} \propto \frac{\cos ^{3} \Psi}{\left(1+3 \sin ^{2} \Psi\right)^{1 / 2}} \\
S_{y} \propto \cos ^{3} \Psi,
\end{gathered}
$$

where $\Psi$ is the geomagnetic latitude of any point on a given field line. Here $S_{x}$ and $S_{y}$ are respectively the separations between points on a given pair of field lines in the $x$-direction (normal to the field line and in the magnetic meridian plane) and the $y$-direction (normal to both the field line and the meridian plane). For our purposes we require the separation as a function of distance along the field line, $l$, rather than of geomagnetic latitude. The geometry of the dipole field leads to the following relation between the differentials of $l$ and $\Psi$ :

$$
d l=-r_{e} \cos \Psi\left(1+3 \sin ^{2} \Psi\right)^{1 / 2} d \Psi,
$$

where $r_{e}$ is the radial distance of the field line in the equatorial plane of the dipole. Integration of this equation leads to

$$
\begin{aligned}
& l=\frac{r_{e}}{2 \sqrt{3}}\left[\sqrt{3} \sin \Psi_{0}\left(1+3 \sin ^{2} \Psi_{0}\right)^{1 / 2}\right. \\
& \quad-\sqrt{3} \sin \Psi\left(1+3 \sin ^{2} \Psi\right)^{1 / 2} \\
& \left.\quad+\sinh ^{-1}\left(\sqrt{3} \sin \Psi_{0}\right)-\sinh ^{-1}(\sqrt{3} \sin \Psi)\right],
\end{aligned}
$$

where $l$ is the distance measured along the field line from a point with geomagnetic latitude $\Psi_{0}$ to a point with geomagnetic latitude $\Psi$. In what follows we shall measure $l$ from the surface of the earth at a geomagnetic latitude of $65^{\circ}$, corresponding roughly to the location of the auroral zone. $l$ is then given by

$$
\begin{aligned}
l(\mathrm{~km})=10,270[4.152- & \left\{1.732 \sin \Psi\left(1+3 \sin ^{2} \Psi\right)^{1 / 2}\right. \\
& \left.\left.+\sinh ^{-1}(1.732 \sin \Psi)\right\}\right]
\end{aligned}
$$

and is illustrated in figure 3 . The separation between neighboring field lines unit distance apart on the earth in the $x$ - and $y$-directions is given by

$$
\begin{aligned}
S_{x} & =\frac{24.58 \cos ^{3} \Psi}{\left(1+3 \sin ^{2} \Psi\right)^{1 / 2}} \\
S_{y} & =13.25 \cos ^{3} \Psi .
\end{aligned}
$$

A sinusoidal electrostatic-field component of spatial wavelength $\lambda$ in the ionosphere now has spatial wavelengths $\lambda_{x}$ and $\lambda_{y}$ in the $x$ - and $y$-directions in the magnetosphere, where $\lambda_{x, y}=\lambda S_{x, y}$. The adopted invariance of $\sigma_{m}$ with altitude implies that $\rho=0$ in (13) and (14), and the term $8 \pi^{2} / \lambda^{2}$ in the same equations must be replaced by $4 \pi^{2}\left(\lambda_{x}^{-2}+\lambda_{y}^{-2}\right)$. 


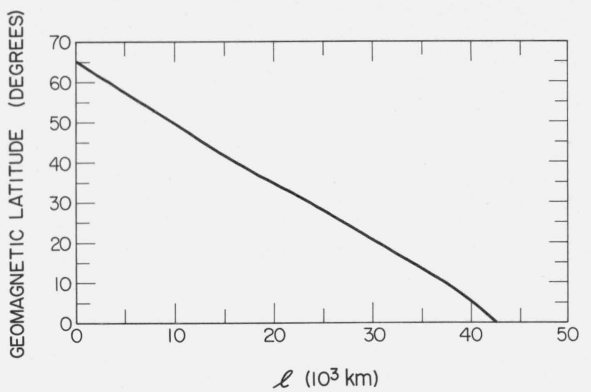

FiguRE 3. Distance (1) measured from the surface of the earth along the dipole field line originating at geomagnetic latitude $65^{\circ}$.

For the purposes of computation, the magnetosphere is divided into a series of slabs, in each of which $\lambda_{x}$ and $\lambda_{y}$ are assumed to be constant, with discontinuous changes at the interfaces. Each slab now has unique values for $\alpha$ and $\beta$ in (14), and $Z$ and $L$ can be found by matching boundary conditions across the interfaces. This model is open to question, since a correct matching across the slab interfaces can be achieved only at a limited number of points, and not over the entire $x-y$ plane. Proper matching could be achieved only if the number of slabs used were infinite, in which case the variation of wavelength with height would be continuous. In practice a model consisting of 22 slabs was used, test computations having shown that the results differed negligibly from those of models using as many as 150 slabs, and thus by reasonable extrapolation from those of a quasicontinuous model.

In the case of the ionosphere, the boundary conditions adopted were continuity of the potential, $\varphi$, and of the longitudinal electrostatic field, $-(\partial \varphi / \partial \zeta)$.

In the case of the magnetosphere, however, the decrease of current density with height introduced by the divergence of the field lines makes this latter boundary condition physically unsatisfactory since it would lead to a discontinuity in longitudinal current flow across the interfaces. A more desirable condition is the continuity of total current, i.e., of the quantity $-S_{x} S_{y}(\partial \varphi / \partial \zeta)$, and the results to be described have been obtained using this condition together with the continuity of the potential in the magnetosphere. The condition expressed in (18b) is thus replaced by

$$
\begin{aligned}
S_{m}\left[\alpha_{m} P_{m} \exp \right. & \left\{\alpha_{m}\left(\zeta-\zeta_{m-1}\right)\right\} \\
+ & \left.\beta_{m} Q_{m} \exp \left\{\beta_{m}\left(\zeta-\zeta_{m-1}\right)\right\}\right] \\
& =S_{m+1}\left[\alpha_{m+1} P_{m+1}+\beta_{m+1} Q_{m+1}\right],
\end{aligned}
$$

where the $S$ 's are the appropriate values of the product $S_{x} S_{y}$.

\section{Method of Calculation}

The numerical calculations consist of the following steps:

(1) The altitude range of interest is divided up into a large number of slabs. In those slabs lying above $500 \mathrm{~km}$ altitude the conductivity $\sigma_{m}$ is assumed to be constant, but the spatial wavelengths of the electrostatic-field components increase with altitude. In those slabs below $500 \mathrm{~km}$, the spatial wavelength is constant with altitude, but the conductivity $\sigma_{m}$ obeys (9).

(2) The conductivities are computed at the interfaces between the slabs in the ionosphere.

(3) The reduced depth, $\zeta_{m}$, of each interface below the source level is found by stepwise numerical integration of $(6 c)$.

(4) The value if $\rho$ is found for each slab below 500 $\mathrm{km}$ from the computed conductivities at the top and bottom of the slab.

(5) The appropriate values of $\alpha$ and $\beta$ are found for each slab using (14) and assuming a value for the spatial wavelength, $\lambda$, of the electrostatic-potential within the ionosphere. The wavelengths within the magnetosphere are found from the divergence of the dipole magnetic-field lines as described above.

(6) The values of $P$ and $Q$ for each slab are found by solution of the simultaneous equations (17), (18), and (19) below $500 \mathrm{~km}$, replacing (18b) by (44) above $500 \mathrm{~km}$.

(7) The transmission factor, $Z$, and the longitudinal field-strength ratio, $L$, are found from (20) and (23).

In practice, 67 slabs were used, 22 within the magnetosphere (above $500 \mathrm{~km}$ ), and 45 within the ionosphere (below $500 \mathrm{~km}$ ). The region from $500 \mathrm{~km}$ to 50 $\mathrm{km}$ was divided into 44 slabs of $10 \mathrm{~km}$ thickness, and the final slab consisted of the region below $60 \mathrm{~km}$. The COSPAR International Reference Atmosphere (CIRA, 1961) was adopted as a model for the neutral atmosphere below $500 \mathrm{~km}$. The source level, at which the electrostatic potential originates, was taken as the equatorial plane, which lies $42,560 \mathrm{~km}$ above the surface of the earth, measured along the field line originating at $65^{\circ}$ geomagnetic latitude. The calculations were carried out on a digital computer.

\section{Results}

The results of the calculations are summarized in figures 4-11, and will be described briefly in this section. All values of the spatial wavelength, $\lambda$, are to be understood as pertaining to the ionosphere. The actual wavelength at the source is considerably greater due to the divergence of the magnetic field lines.

The potential transmission factor, $Z$, is shown in figures 4 and 5 for daytime and nighttime conditions respectively, and for a variety of values of the spatial wavelength, $\lambda$. As would be expected intuitively, the downward transmission of electrostatic potentials is considerably less efficient by day than by night, due to the increased conductivity of the ionosphere, and 


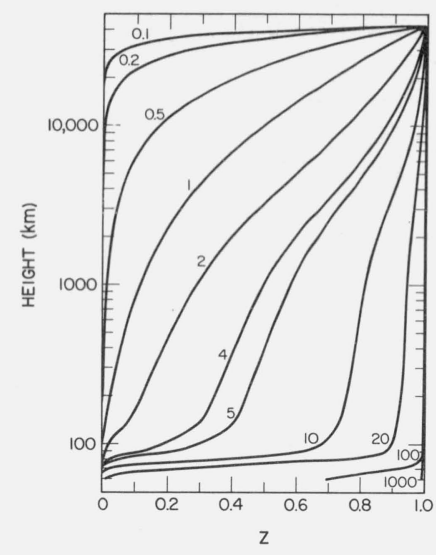

FIgURE 4. The potential transmission factor (Z) as a function of altitude for daytime conditions.

The labels on the curves refer to the spatial wavelength $(\lambda)$ in kilometers.

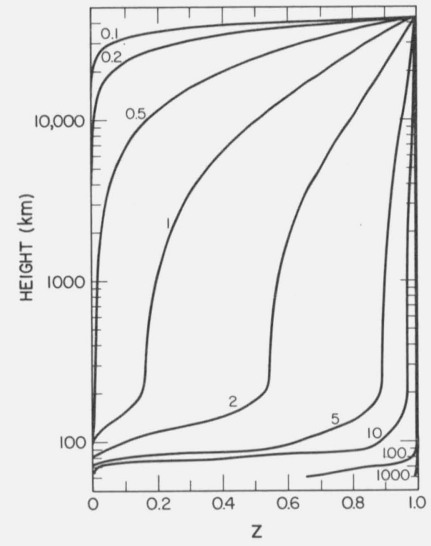

FIGURE 5. The potential transmission factor (Z) as a function of altitude for nighttime conditions.

The labels on the curves refer to the spatial wavelength $(\lambda)$ in kilometers.

its consequently increased 'short-circuiting' action through the Pedersen currents. For both day and night conditions, however, it can be concluded that potential variations with wavelengths less than about $1 \mathrm{~km}$ in the ionosphere are not likely to be transmitted from the outer magnetosphere, whereas those with wavelengths greater than about $10 \mathrm{~km}$ can be transmitted very efficiently. Thus potential variations over a global scale, such as those envisaged by Axford and Hines [1961] as arising from the convection of the magnetosphere, will appear in the ionosphere, and the motion of the ionospheric plasma will duplicate that of the magnetospheric plasma. The small-scale irregularities invoked by many authors to explain auroral radar reflections, however, are unlikely to arise in this way unless the corresponding potential variations in the magnetosphere are assumed to be of extremely large amplitude. Similarly, the irregularities thought to be responsible for radio-star 'fadeouts' [Little et al., 1962; Moorcroft, 1963; Flood, 1963] are unlikely to arise in this way, since their spatial wavelength is thought to be considerably less than $1 \mathrm{~km}$. The ionospheric irregularities responsible for normal radio-star scintillation, however, have scale-sizes that are typically of the order of a few kilometers, and may well arise from turbulent electrostatic fields transmitted downward from the outer magnetosphere, being attenuated in the process by a factor of 1 to 5 . It is evident from figures 4 and 5 that the attenuation in the $E$ region would be considerably greater than in the $F$ region, so that this mechanism would not be expected to form such pronounced $E$-region irregularities, especially during daytime. Observations (e.g., Yeh and Swenson [1964]) have shown that most, if not all, of the irregularities responsible for scintillation are indeed located in the $F$ region. This point will be discussed further in the following section.

The relative efficiency of transfer to the $F$ region and the $E$ region is illustrated in figures 6 and 7 ,

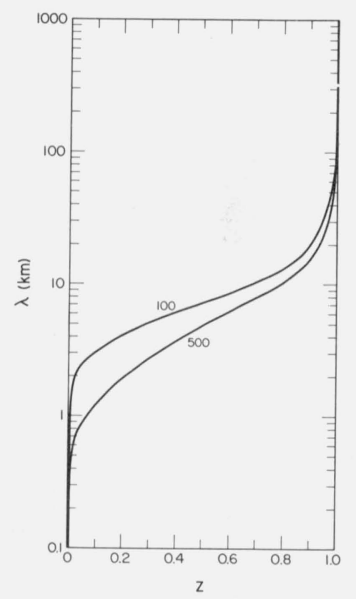

FIGURE 6. The potential transmission factor (Z) as a function of $\lambda$ for daytime conditions.

The curves refer to altitudes of $500 \mathrm{~km}$ and $100 \mathrm{~km}$.

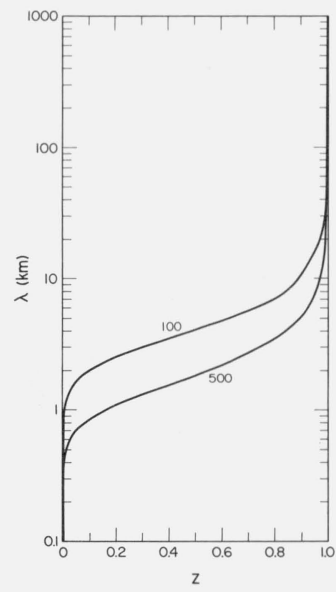

Figure 7. The potential transmission factor (Z) as a function of $\lambda$ for nighttime conditions.

The curves refer to altitudes of $500 \mathrm{~km}$ and $100 \mathrm{~km}$. 
which show $Z$ as a function of $\lambda$ at $500 \mathrm{~km}$ and at $100 \mathrm{~km}$ during daytime and nighttime conditions respectively. It is evident that electrostatic-field components with wavelengths lying between about $1 \mathrm{~km}$ and $5 \mathrm{~km}$ can be transferred quite efficiently down to $500 \mathrm{~km}$, but would not be expected to appear as low as $100 \mathrm{~km}$.

Figures 8 and 9 show the electrostatic-field component along the magnetic field which must necessarily be associated with the drop in potential illustrated in the previous diagrams. The parameter $L$ is shown as a function of altitude for a variety of wavelengths for daytime and nighttime conditions. It will be recalled that $L$ is defined as the ratio of the longitudinal electrostatic field to the source-level potential at the top of the magnetic field line. In particular, if the amplitude of the sinusoidal potential variation at the source level is $\varphi_{0}$, then the maximum longitudinal electrostatic fields appear along the field line whose source potential is $\varphi_{0}$, and are given by $-(\partial \varphi / \partial z)=L \varphi_{0}$.

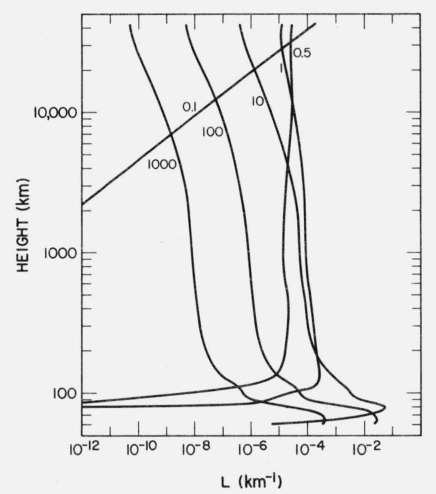

FIGURE 8. The longitudinal-field parameter (L) as a function of altitude for daytime conditions.

The labels on the curves refer to the spatial wavelength $(\lambda)$ in $\mathrm{km}$.

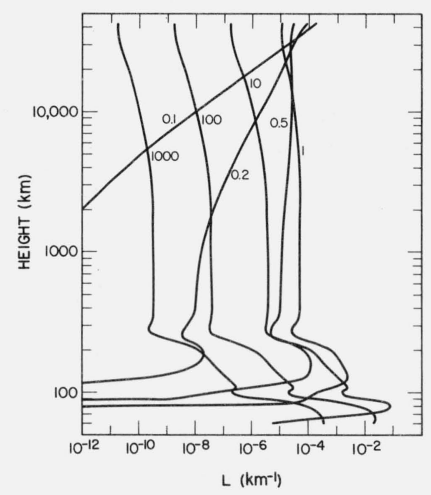

FIGURE 9. The longitudinal-field parameter (L) as a function of altitude for nighttime conditions.

The labels on the curves refer to the spatial wavelength $(\lambda)$ in $\mathrm{km}$.
In the case of the smallest scale-sizes, these fields are confined to the magnetosphere and upper ionosphere, since the electrostatic potential vanishes before reaching the $F$ region. For the larger scalesizes, $L$ increases rather rapidly below about $200 \mathrm{~km}$ due to the increasing flow of Pedersen current and reaches a maximum well below the $E$ region. The actual form of the height variation in this region will depend critically on the model adopted for the conductivity of the $D$ region and the lower atmosphere; the model chosen here is not thought to be particularly accurate below $100 \mathrm{~km}$, and detailed consideration of conditions at these altitudes will be deferred.

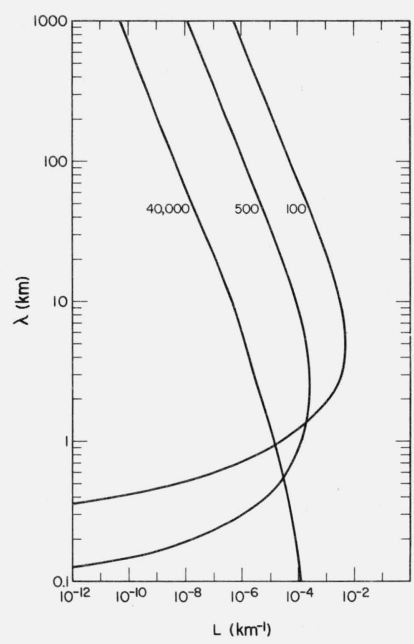

FIGURE 10. The longitudinal-field parameter (L) as a function of $\lambda$ for daytime conditions at altitudes of $40,000 \mathrm{~km}, 500 \mathrm{~km}$, and $100 \mathrm{~km}$.

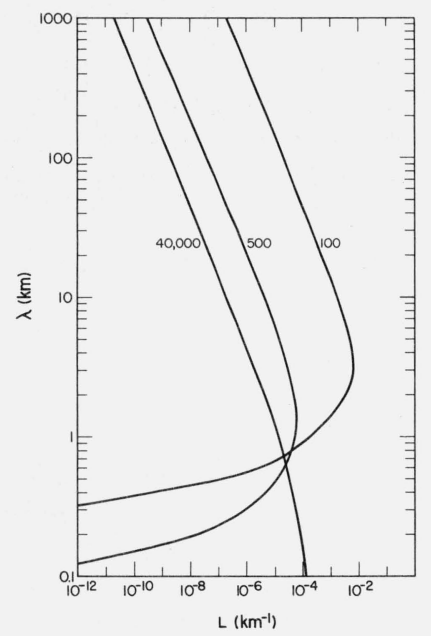

FIGURE 11. The longitudinal-field parameter (L) as a function of $\lambda$ for nighttime conditions at altitudes of $40,000 \mathrm{~km}, 500 \mathrm{~km}$, and $100 \mathrm{~km}$. 
Since the motion of an electron along the direction of the magnetic field is unaffected by the presence of the magnetic field, these electrostatic fields may be expected to be important from the point of view of electron acceleration. In particular, those electrons in the high-energy tail of the thermal distribution function may well take part in the well-known 'runaway' phenomenon of plasma physics [e.g., Rose and Clark, 1961], gaining an energy comparable with the entire potential drop between the top of the magnetic field line and the altitude at which they make their first collision with a neutral particle. This suggestion will be discussed further, with some numerical estimates, in the following section.

The variation of $L$ with $\lambda$ is illustrated in figures 10 and 11 for altitudes of $40,000 \mathrm{~km}$ (near the top of the magnetic field line), $500 \mathrm{~km}$, and $100 \mathrm{~km}$. At $500 \mathrm{~km} L$ is a maximum for a wavelength of $3 \mathrm{~km}$ during the day and $1.5 \mathrm{~km}$ during the night. At $100 \mathrm{~km}$ altitude the corresponding values of $\lambda$ are $5 \mathrm{~km}$ and $3 \mathrm{~km}$. Thus the maximum ratios of longitudinal electrostatic field to source potential are attained in the ionosphere for spatial wavelengths of a few kilometers, comparable with the scale-sizes of the radio-starscintillation irregularities. For a given amplitude of source potential, figures 8 and 9 show that the maximum total potential drop down to the $F$ region is attained for values of $\lambda$ less than $1 \mathrm{~km}$. However, as we shall see in the following section, the actual value of the source-potential amplitude is likely to be much smaller for these wavelengths than for the larger scale-sizes.

\section{Discussion}

It is of some interest to make a few numerical estimates of the sizes of the effects we have been discussing. As was mentioned in the initial discussion of the model, any arbitrary pattern of turbulent electrostatic fields can be decomposed into Fourier components, and we have treated the components of different wavelength separately. The downward mapping process for an arbitrary pattern could be examined by recombining the Fourier components after multiplication by the appropriate value of $Z$ at any desired level in the ionosphere. In this discussion, however, we shall again confine ourselves to the consideration of individual sinusoidal components.

A number of assumptions have been made in order to simplify the analysis; for instance, the magnetic field within the ionosphere has been assumed to be vertical, the conductivities have been assumed to be constant above $500 \mathrm{~km}$, and horizontal variations in conductivity have not been permitted. Even with these assumptions, it is felt that the results have some quantitative significance. A more complete analysis would, of course, be highly desirable, but is outside our present scope.

The large-scale magnetospheric convection system of Axford and Hines [1961] can be thought of as a Fourier component with wavelength of the order of $1000 \mathrm{~km}$ in the ionosphere, and with a source-poten- tial amplitude, $\varphi_{0}$, of about $10,000 \mathrm{~V}$ (derived from the observed ionospheric drift speeds of the order of 300 $\mathrm{m} \mathrm{sec}{ }^{-1}$ and the known magnetic-field strength in the ionosphere). The results shown in figures 8 and 9 then indicate that the maximum longitudinal electrostatic field associated with this convection system would be of order $10^{-8}$ to $10^{-10} \mathrm{~V} / \mathrm{m}^{-1}$. Even if applied along the entire length of the magnetic field line, such potential gradients could only supply electrons with energies comparable with, or an order of magnitude greater than, their thermal energy.

An estimate of the size of the source-potential amplitude associated with any component can be obtained from the magnitudes of ionospheric drift speeds. The electrostatic field, $E$, associated with a drift speed $v$ is given approximately by $v B$. Also the maximum electrostatic field arising from a sinusoidal horizontal variation of potential of wavelength $\lambda$ is given by $(2 \pi / \lambda) \varphi_{0}$, where $\varphi_{0}$ is the source-potential amplitude. Equating these, we have

$$
\varphi_{0}=\frac{\lambda v B}{2 \pi}
$$

The value of $B$ in the ionosphere can be taken as $3 \times 10^{-5} \mathrm{~Wb} / \mathrm{m}^{-2}$; appropriate values for $v$ are largely a matter of guesswork, since $v$ refers to the turbulent component of the drift speed, rather than its actual total magnitude. For our present purpose it might be reasonable to adopt values of about $100 \mathrm{~m} \mathrm{sec}^{-1}$ and $1 \mathrm{~km} \mathrm{sec}^{-1}$, the latter to be regarded as an upper limit, since it is comparable with the total drift speeds observed under fairly disturbed conditions. $\varphi_{0}$ can then be taken as lying in the range $0.5 \lambda$ to $5 \lambda$, where $\varphi_{0}$ is measured in volts and $\lambda$ in kilometers.

The longitudinal electrostatic field is given by the product $L \varphi_{0}$, and is a maximum for wavelengths of the order of $10 \mathrm{~km}$. Taking the upper limit for the source potential given above, we find $\varphi_{0}=50 \mathrm{~V}$ at $\lambda=10 \mathrm{~km}$, and figures 8 and 9 then give a longitudinal field of about $5 \times 10^{-7} \mathrm{~V} \mathrm{~m}^{-1}$ throughout the magnetosphere. Thus the total potential drop along a magnetic field line from the equatorial plane to the top of the ionosphere is of the order of $20 \mathrm{~V}$, a value in excess of the ionization potential of the atmospheric constituents. When we allow the possibility of an individual electron making several up-and-down trips in a region containing irregularities, it is apparent that these longitudinal electrostatic fields may well provide a source of energy for the ionization of the $F$ region.

\section{Generation of the Fields}

We have considered the distribution of electrostatic potentials necessary to provide a divergencefree flow of current transverse to the magnetic field in the ionosphere and along the magnetic field lines connecting the ionosphere to the magnetosphere, but we have not yet mentioned the remaining current component required to close the loop. Under certain 
conditions (identical spatial variations of conductivity profile in both hemispheres), the completion of the current loop could presumably be provided by the ionosphere in the conjugate hemisphere without invoking any contribution from transverse currents within the magnetosphere. In general, however, such a contribution must be supplied, and here we shall briefly discuss the nature of this current, which is intimately connected with the mechanism of generation of the electrostatic fields.

Any driving force acting on the magnetospheric plasma, such as the viscous force of the AxfordHines theory, can be represented by a body force F acting on unit volume. Such a force acting on a highly ionized plasma causes the ions and electrons to drift in opposite directions, given respectively by the directions of the vectors $\mathbf{F} \times \mathbf{B}$ and $-\mathbf{F} \times \mathbf{B}$. The resulting charge separation sets up an electrostatic field $\mathbf{E}$ in the direction of $-\mathbf{F} \times \mathbf{B}$, which in turn causes a drift in the direction of $\mathbf{E} \times \mathbf{B}$, i.e., in the direction of the driving force $F$. If there were no ionospheric currents to consider, the field $\mathbf{E}$ would grow to a point at which the drift speed $E / B$ equalled the solar wind speed. There would then be no net body force, and the 'polarizing' current would cease to flow, having achieved its purpose. In the presence of ionospheric currents, however, the polarizing current must flow continuously to close the ionospheric-current loop, and enough resultant body-force must remain to generate this current. In other words, the magnetospheric plasma will accelerate only to a point at which the difference between its speed and that of the solar wind is sufficient that the resultant body force generates the required current. In this steady-state condition the current will exert a body force $\mathbf{j} \times \mathbf{B}$ on the medium, which will exactly balance the resultant driving force. The necessary current densities, $\mathbf{j}$, in the magnetosphere are quite small. For example, an electrostatic field of $1.5 \times 10^{-2} \mathrm{~V} / \mathrm{m}^{-1}$ applied in the meridian plane throughout the daytime ionosphere will give an integrated Pedersen current of about $1.3 \times 10^{-2} \mathrm{~A} / \mathrm{m}$ of longitude. If we assume that the driving force is applied over a length of some $10,000 \mathrm{~km}$ at the outer edge of the field line, and allow a dilution factor of about 10 due to the divergence of the field lines, this current can be closed by a current density of about $1.3 \times 10^{-10} \mathrm{~A} \mathrm{~m}^{-2}$ in the outer magnetosphere. If the electron density there is in the range $10^{8}$ to $10^{9} \mathrm{~m}^{-3}$, this current density can be provided by a drift of ions and electrons in opposite directions with speeds of the order of 1 to $10 \mathrm{~m} \mathrm{sec}^{-1}$, which is extremely small in comparison with the drift speeds normal to $\mathbf{E}$ and $\mathbf{B}$ of about $10 \mathrm{~km} \mathrm{sec}-1$ required by the observed ionospheric drift speeds of the order of $1 \mathrm{~km} \mathrm{sec}-1$. Thus there appears to be no conceptual difficulty in visualizing the closing of the current loops by way of 'polarizing' currents transverse to the magnetic field in the outer magnetosphere. The full details of this process will not, however, be considered here.

\section{Conclusion}

We have attempted to examine quantitatively the ionospheric effects of electrostatic fields generated in the outer magnetosphere by motions of the plasma. It has been shown that the existence of small-scale 'turbulence' in the magnetospheric motions may have far-reaching effects in the ionosphere, leading to the generation of irregularities in electron density, primarily in the $F$ region, and possibly also to the acceleration of electrons to energies in excess of ionization potentials.

As was mentioned in the introduction, however, the generation of $F$-region irregularities by this mechanism requires the prior existence of gradients of electron density at the appropriate heights, and this point has not been discussed in the present paper. A further factor that influences the formation of irregularities is the lifetime of an individual electron; for example, an irregularity of one percent deviation from the background electron density can form in this way only if the average lifetime of an electron is sufficiently long that it can be transported by the random fields between points whose background electron density differs by one percent. The height of occurrence of irregularities is thus likely to be determined by the most favorable combination of the various factors.

The mechanisms involved appear to warrant further investigation, and in particular it would appear highly desirable to consider the effects of small-scale variations of conductivity in the horizontal direction, which have been ignored in the present discussion. During an auroral display, for example, the fine structure observed in the visible light must be accompanied by corresponding horizontal fine structure in the ionospheric conductivity. Even if no turbulent structure is present in the magnetospheric plasma motions, horizontal fine structure in the conductivity must give rise to horizontal variations in Pedersen-current flow, and corresponding longitudinal electrostatic fields associated with the large-scale convection of the magnetosphere. A discussion of these effects will be deferred. It would also be desirable to investigate the conditions under which appreciable numbers of 'runaway' electrons might be generated, and the possibility that these may contribute to the formation of the outer radiation belt.

Yet another feature of the actual magnetosphere has been ignored, and merits further investigation. We have treated the geomagnetic field as though it terminated abruptly in the equatorial plane; in fact any given field line returns to the earth in the opposite hemisphere, and may there encounter an entirely different ionospheric conductivity profile. Thus any electrostatic field generated near the equatorial plane is faced with two 'circuits' in parallel, and the consequences appear to be well worth investigating. 


\section{References}

Akasofu, S.-I., and S. Chapman (1961), The ring current, geomagnetic disturbance, and the Van Allen radiation belts, J. Geophys. Res. 66, 1321-1350.

Axford, W. I., and C. O. Hines (1961), A unifying theory of highlatitude geophysical phenomena and geomagnetic storms, Can. J. Phys. 39, 1433-1464.

Baker, W. G., and D. F. Martyn (1953), Electric current's in the ionosphere. I. The conductivity. Phil. Trans. Roy. Soc. London, A246, 281-294.

Chapman, S. (1956), The electrical conductivity of the ionosphere: a review, Nuovo Cimento, Suppl. Ser. 10, 4, 1385-1412.

Chapman, S., and T. G. Cowling (1939), The Mathematical Theory of Non-Uniform Gases (Cambridge University Press, Cambridge).

CIRA (1961) COSPAR International Reference Atmosphere (NorthHolland Publishing Company, Amsterdam)

Cowling, T. G. (1945), The electrical conductivity of an ionized gas in a magnetic field, with applications to the solar atmosphere and the ionosphere, Proc. Roy. Soc. London, A183, 453-479.

Dagg, M. (1957), The origin of the ionospheric irregularities responsible for radio-star scintillations and spread $F$. II. Turbulent motion in the dynamo region, J. Atmospheric Terrest. Phys. 11, 139-150.

Dougherty, J. P. (1959), Magnetohydrodynamics of the small-scale structure of the $F$ region, J. Geophys. Res. 64, 2215-2216.

Farley, D. T. (1959), A theory of electrostatic fields in a horizontally stratified ionosphere subject to a vertical magnetic field, J. Geophys. Res. 64, 1225-1233.

Farley, D. T. (1960), A theory of electrostatic fields in the ionosphere at non-polar geomagnetic latitudes, J. Geophys. Res. 65, 869-877.

Farley, D. T. (1961), Discussion of paper by John R. Spreiter and Benjamin R. Briggs on 'Theory of electrostatic fields in the ionosphere at polar and middle geomagnetic latitudes,' J. Geophys. Res. 66, 3956-3957.
Flood, W. A. (1963), A study of radio star fadeouts and their application to radar resolution, J. Geophys. Res. 68, 4129-4140.

Hines, C. O. (1963), The energization of plasma in the magnetosphere: hydromagnetic and particle-drift approaches, Planetary Space Sci. 10, 239-246.

Little, C. G., G. C. Reid, E. Stiltner, and R. P. Merritt (1962), An experimental investigation of the scintillation of radio stars observed at frequencies of 223 and 456 megacycles per second from a location close to the auroral zone, J. Geophys. Res. 67, 17631784.

Moorcroft, D. R. (1963), Radio star fadeouts on phase-switching interferometer records, J. Geophys. Res. 68, 111-115.

Rose, D. J., and M. Clark (1961), Plasmas and Controlled Fusion (M.I.T. Press and John Wiley \& Sons, New York, N.Y.).

Smith, R. L. (1961), Properties of the outer ionosphere deduced from nose whistlers, J. Geophys. Res. 66, 3709-3716.

Smythe, W. R. (1939), Static and Dynamic Electricity, lst ed. (McGraw-Hill Book Co., Inc., New York 243).

Spreiter, J. R., and B. R. Briggs (1961), Theory of electrostatic fields in the ionosphere at polar and middle geomagnetic latitudes, J. Geophys. Res. 66, 1731-1744.

Wait, J. R., and A. M. Conda (1958), On the measurement of ground conductivity at VLF, IRE Trans. Ant. Prop. AP-6, 273-277.

Yeh, K. C., and G. W. Swenson, Jr. (1964), F-region irregularities studied by scintillation of signals from satellites, Radio Sci. J. Res. NBS 68D, No. 8, 881-894.

(Paper 69D6-515) 